\title{
Strategy switch costs in arithmetic problem solving
}

\author{
Patrick Lemaire and Mireille Lecacheur \\ CNRS and Université de Provence, Marseille, France
}

\begin{abstract}
Three experiments tested whether switching between strategies involves a cost. In three experiments, participants had to give approximate products to two-digit multiplication problems (e.g., $47 \times 76$ ). They were told which strategy to use (Experiments 1 and 2) or could choose among strategies (Experiment 3 ). The participants showed poorer performance when they used different strategies on two consecutive trials than when they used the same strategy. They also used the same strategy over two consecutive problems more often than they used different strategies. These effects, termed strategy switch costs, were found when the participants executed the easiest strategy and when they solved easy problems. We discuss possible processes underlying these strategy switch costs and the implications of these strategy switch costs for models of strategy choices.
\end{abstract}

One of the hallmarks of human cognition is that participants use several strategies when they accomplish cognitive tasks (Siegler, 2007). This multiple-strategy use permits one to alternate flexibly between different strategies and to adapt to the demands of problems and situations. Two central issues with regard to cognitive strategies concern how we choose among strategies for solving each problem and how we execute a strategy once it has been selected. The present study contributes to these issues by showing that participants obtain poorer performance when they use different strategies on two consecutive trials than when they use the same strategy and tend to repeat the same strategy over successive problems.

Previous findings on cognitive strategies showed that both strategy choices and strategy execution are influenced by problem and strategy characteristics, as well as by participants' skills, working memory capacities, and age. For example, in arithmetic, participants tend to retrieve correct solutions to problems like $3 \times 4$ directly from memory when retrieval is accurate and fast and to use counting on problems like $7 \times 8$ when retrieval is not accurate (e.g., Campbell \& Xue, 2001; LeFevre, Bisanz, Daley, Buffone, \& Sadesky, 1996). As another example, in episodic memory, participants tend to use mental imagery more often to memorize concrete words and mental rehearsal to store more abstract words (e.g., Dunlosky \& Hertzog, 2001).

Computational models of strategy choices (e.g., Lovett \& Anderson's [1996] ACT-R model; Lovett \& Schunn's [1999] RCCL model; Payne, Bettman, \& Johnson's [1993] adaptive decision maker model; Rieskamp \& Otto's [2006] SSL model; and Siegler \& Arraya's [2005] SCADS* model) share several core assumptions regarding how we choose among strategies. All these models propose that choosing among multiple strategies crucially involves associative mechanisms such as activating the relative costs/ benefits of each strategy and selecting the strategy that works best for a given problem on the basis of problem and strategy characteristics. All models also assume that strategies including fewer and/or simpler procedures (e.g., retrieving the correct solution of arithmetic problems like $3 \times 4$ directly from memory) are easier to execute than strategies including more and/or more complex procedures (e.g., adding 3 four times). These assumptions have proven sufficient to account for most findings on strategy choices and strategy execution.

However, several strategy phenomena are not accounted for by models of strategy choices. For example, it is unknown why some groups of participants, such as older adults (see, e.g., Lemaire \& Arnaud, 2008), sometimes use fewer strategies even when they know and can use all available strategies. Also, it is unknown why problem features and relative strategy efficacy together never fully account for strategy choices or why participants are sometimes biased in strategy choices (i.e., they continue to use a strategy when an alternative is faster and/or more accurate; e.g., Lemaire \& Siegler, 1995; Lovett \& Anderson, 1996; Luwel, Verschaffel, Onghena, \& De Corte, 2003; Siegler \& Lemaire, 1997). Such an Einstellung, or inertia, effect (in which people habitually apply a strategy that has successfully worked out even though there may be a better and more efficient strategy available; Luchins, 1942; Rieskamp \& Otto, 2006) suggests that adopting a strategy is not just a product of considering problem and strategy characteristics. The present study investigated the possibility that, at least in some contexts, strategy selection and strategy execution on a given problem are influenced by the strategy that was applied to the previ- 
ous problem. More specifically, we tested the hypothesis that switching strategy from one trial to the next incurs cognitive cost. Such strategy switch costs would be seen if participants have poorer performance when they switch strategies from one trial to the next than when they repeat the same strategy.

The hypothesis that strategy use and strategy selection on a given trial are influenced by the strategy that was executed on the preceding trial was inspired by findings from task-switching experiments (see Monsell, 2005, for an overview). In task-switching experiments, participants are presented with stimuli that afford two (or more) tasks and are instructed by cues as to which task is relevant on each trial. Participants either repeat the same task on two consecutive trials or switch tasks. Usually, performance is better when participants repeat the same task than when they accomplish two different tasks (e.g., Meiran, 1996; Meiran, Chorev, \& Sapir, 2000; Rogers \& Monsell, 1995; Sohn \& Carlson, 2000). Although interpretations of task switch costs are still debated in the literature, some authors (e.g., Monsell, 2005; Monsell, Taylor, \& Murphy, 2001; Monsell, Yeung, \& Azuma, 2000; Yeung \& Monsell, 2003) have assumed that they result, in part, from executive control processes (i.e., activating the currently relevant task and deactivating the just-accomplished task). Other authors (e.g., Allport \& Wylie, 2000; Wylie \& Allport, 2000; Wylie, Javitt, \& Foxe, 2003a, 2003b, 2006) have assumed that they result from task set inertia or interference (competition) with persisting, task-related activity of the previous trial. The two types of mechanisms - interference and executive control - may combine to produce task switch costs (e.g., Sohn \& Anderson, 2001). Finding the equivalent of task switch costs (i.e., strategy switch costs) would suggest that strategy choices and strategy execution involve executive control and/or interference processes. Such findings would also generalize findings from the task-switching literature to strategy switching.

Task switching and strategy switching may be viewed as similar since, at a very general and abstract level, both entail switching between two mental entities of equal or unequal difficulty. Moreover, strategies are task components. Finally, both strategy and task switching are involved in maintaining, coordinating, and selecting goaldirected actions and controlling interference. Therefore, strategy switch costs may be found, like task switch costs, and may vary with the same parameters as those for task switch costs (e.g., type of strategies). However, they are also different. In task switching, people switch from two different goals and use two different sets of procedures on each item (e.g., judging whether a stimulus is a square or a circle vs. judging whether this stimulus is blue or green). In strategy switching, people have the same goal (e.g., finding the correct product of a multiplication problem) on each problem but switch between two sets of procedures to accomplish this goal (e.g., retrieving the solution directly from memory or using a counting strategy, such as doing $4+4+4$ to solve $3 \times 4$ ). Moreover, strategy choices depend on problem features, such that each individual problem activates one strategy automatically, whereas tasks (at least tasks used in many task-switching experiments) do not have specific and privileged associations with problem features (e.g., the number 6 is both even and larger than 5; it is not "more even" and "less five," whereas retrieval strategy is more often used than counting with problems like $2+3$ in arithmetic-problemsolving tasks). Finally, in contrast to strategy experiments, most tasks used in task-switching experiments are very simple tasks, since they involve very few steps to be accomplished (e.g., determining whether a digit is smaller or larger than 5 involves fewer processes than does solving $37 \times 69$ ). These differences between strategy and task switching may result in different switch costs when participants switch between tasks versus between strategies. If there are any, strategy switch costs may not be influenced by the same variables as task switch costs, or to different extents. It could even be that switching between strategies entails no switching costs.

In the present experiments, strategy switch costs were investigated in the context of computational estimation tasks (i.e., determining the approximate result of an arithmetic problem like $64 \times 89$ without calculating the exact answer). Previous studies showed that participants use several rounding strategies to accomplish these computational estimation tasks (e.g., rounding both operands down to the closest smaller decades, rounding both operands up to the closest larger decades, rounding one operand down and the other up to the closest decades, rounding with or without adjustments; e.g., LeFevre, Greenham, \& Waheed, 1993; Lemaire, Arnaud, \& Lecacheur, 2004; Lemaire, Lecacheur, \& Farioli, 2000). In all the experiments, the participants saw trials with two successive problems each. The participants were cued as to which strategy to use to solve each problem on each trial in Experiments 1 and 2 and which strategy to use on the first problem only (and were free to choose among strategies on the second problem) in Experiment 3. We predicted that the participants would be faster and/or more accurate when they used the same strategy on two consecutive problems than when they used different strategies (Experiments 1 and 2) and that they would tend to repeat the same strategy on two consecutive problems (Experiment 3).

\section{EXPERIMENT 1 Strategy Switch Costs During Strategy Execution}

In this experiment, we tested strategy switch costs during strategy execution in the context of a computational estimation task. Strategy switch costs would be seen in differences in participants' performance when they used the same strategy on two consecutive trials as compared with when they used different strategies.

The participants had to give approximate products to two-digit multiplication problems (e.g., $47 \times 76$ ). On each problem, they were told which strategy to use, rounding down or rounding up. The rounding-down strategy involved rounding both operands down to the closest decades (e.g., $70 \times 40$ to solve $78 \times 43$ ), and the rounding-up strategy involved rounding both operands up to the closest decades (e.g., $80 \times 50)$. The problems to 
be solved could be small-unit problems (i.e., unit digits of both operands were smaller than 5; e.g., $42 \times 73$ ) or large-unit problems $(47 \times 58)$. These two strategies and two types of problems were tested because previous works had shown that participants' performance was poorer with the rounding-up strategy and with the large-unit problems (e.g., LeFevre et al., 1993; Lemaire et al., 2004; Lemaire et al., 2000). As was discussed at length by Lemaire et al. (2004), the former is harder because participants have to temporarily store operands in working memory before incrementing them and to calculate on larger operands. In the rounding-down strategy, participants do not need to store operands in working memory, since the operands are displayed on the computer screen while being processed; they do not need to increment operands, and they calculate on smaller numbers.

In this experiment, we also wanted to determine whether strategy switch costs interact with problem and strategy characteristics. This was motivated by the findings that task switch costs interact with task difficulty. Indeed, several authors had found that task switch costs are larger when participants switch from a hard to an easy task than when switching from an easy to a hard task (e.g., Allport, Styles, \& Hsieh, 1994; Yeung \& Monsell, 2003). If task switch costs generalize to strategy switch costs, we should find the largest strategy switch costs when participants switch from a hard to an easy strategy (i.e., rounding-down strategy) and when they solve an easy problem (i.e., smallunit problems) after solving a hard problem (i.e., large-unit problems).

The participants saw 240 trials. A trial included two successive problems, followed by a series of letters. The participants were asked to solve each problem successively with a given strategy and then to determine whether each series of letters included letters of the same type (only vowels or only consonants) or letters of different types (vowels and consonants). This task structure was aimed at avoiding the possibility that switch costs on the second problems would be contaminated by carryover effects from previous problems.

Strategy switch costs would be seen if the participants had poorer performance (i.e., latency and accuracy) on the second problem of a trial when they executed different strategies on the two consecutive problems of a trial, as compared with when they executed the same strategy on each problem. Moreover, if these strategy switch costs are modulated by both the type of strategy that people use and the type of problems that people solve, problem (small-unit/ large-unit) $\times$ trial (repeated-strategy/unrepeated-strategy) and strategy (rounding-down/rounding-up) $\times$ trial interactions should be observed. Such interactions would consist of larger strategy switch costs when participants switched from using the rounding-up strategy to using the rounding-down strategy and when solving small-unit problems after solving large-unit problems.

\section{Method}

Participants. Thirty (11 males; mean age, 24 years 2 months; age range, 18.5-28.4) undergraduates from the University of Provence (Marseille, France) received course credit for their participation.
Stimuli. A set of 240 trials was created. Each trial was made of two consecutive two-digit multiplication problems. Each pair of problems was followed by a series of five letters. Half of the fiveletter series included only consonants or only vowels, and half included both types of letters.

Half of the first and second multiplication problems on a given trial were small-unit problems, and the other half were large-unit problems. The unit digits for both types of problems were smaller than 5 (e.g., $32 \times 43$ ) in small-unit problems and larger than 5 (e.g., $28 \times 49)$ in large-unit problems. Following previous findings in arithmetic (see Campbell, 2005, for an overview), we controlled the following factors: (1) No operands had a 0 unit digit (e.g., $40 \times 82$ ); (2) no operands had a 5 unit digit (e.g., $45 \times 82$ ); (3) no digits were repeated within operands (e.g., $44 \times 82)$; $(4)$ no reverse orders of operands were used (e.g., $43 \times 82$ and $82 \times 43$ ); (5) the first operand was larger than the second in half the problems, and vice versa; (6) no operand had its closest decade equal to 0,10 , or 100 ; (7) differences between correct products and estimates were matched across strategies (i.e., mean percentages of deviations were equal when the participants used the rounding-up or the rounding-down strategy on all the problems); (8) small- and large-unit problems had comparable correct products; and (9) rounded operands were never the same across the two problems on a given trial (e.g., if the first problem in a trial was $42 \times 53$, the second problem could not be $41 \times 54)$.

Procedure. The participants were individually tested in two sessions, each of which lasted approximately 45-60 $\mathrm{min}$. The two sessions were at least 7-10 days apart. Before encountering the experimental problems, in the first session, the participants were told that they were going to do computational estimation. Computational estimation was explained as giving an approximate answer to an arithmetic problem that was as close as possible to the correct answer without actually calculating the correct answer. An example was worked out with the participants, who were told the following:

For example, if I have to estimate $78 \times 43$, I can do $70 \times 40$ and give 2,800 as an approximate solution to the problem. I can also do $80 \times 50$, or do anything else that yields an approximate answer. To solve each problem, you will be able to use either of two rounding strategies, rounding down and rounding up. The rounding-down strategy involves rounding both operands down to the closest smaller decades (e.g., $70 \times 40$ to solve $78 \times 43$ ).

The rounding-up strategy involves rounding both operands up to the closest larger decades (e.g., $80 \times 50)$.

Instructions emphasized that the participants should not use any other strategies, should do only the initial rounding up or down, and should do nothing more (i.e., adding or subtracting small amounts) after calculating the product of rounded operands. After 16 practice problems ( 8 with each rounding strategy), all the individuals had no difficulties with either rounding strategy; none of them tried to calculate the exact products. Then the participants practiced the experimental task on 8 trials (each involving two multiplication problems and a series of five letters) for them to get familiarized with the procedure and the structure of each trial. This training session was carried out in both sessions before the experiment started in earnest. In both sessions, the participants solved 120 trials with a break in between each block of 30 problems.

The participants used the same strategy on two consecutive problems for half the trials (repeated-strategy trials) and a different strategy for the other trials (unrepeated-strategy trials). In each condition, half the small-unit problems were solved with the roundingdown strategy, and half with the rounding-up strategy. Similarly, half the large-unit problems were solved with the rounding-up strategy, and half with the rounding-down strategy.

The stimuli were presented in 48-point bold Courier font (black color) in the center of a 14-in. computer screen controlled by a Dell D620 PC. Each trial began with a blank screen for $500 \mathrm{msec}$, followed by a warning signal (*) appearing for $400 \mathrm{msec}$ in the center of the screen. Then the problem and the cue were simultaneously 
displayed on the computer screen. The cue appeared $2 \mathrm{~cm}$ above the problem and, together with the problem, remained on the screen until the participants' response. The letter "B" (standing for bas, or down in French) cued the participants to solve the current problem with the rounding-down strategy, and the letter " $\mathrm{H}$ " (haut, or up in French) prompted the participants to use the rounding-up strategy. After the participants' response, a blank screen was presented for $500 \mathrm{msec}$, followed by a "* " warning signal for $400 \mathrm{msec}$. The second problem of a trial and the cue were then presented. Timing of each response began when the problem and the cue appeared on the screen and ended when the experimenter pressed the space bar of the computer keyboard, the latter event occurring as soon as possible after the participants' responses. A blank screen followed the participants' response for $500 \mathrm{msec}$. Then "* " appeared for $400 \mathrm{msec}$, followed by five letters (e.g., aeiou). A blank screen was finally displayed for $1,000 \mathrm{msec}$ before the next trial started. The participants were asked to calculate aloud as a control showing that they were using the strategy that they were supposed to use (which they did on $100 \%$ of the problems). The experiment was controlled by E-Prime software, and the experimenter was not informed about the goal of the experiments, the factors that we manipulated, and our hypotheses.

\section{Results}

The results were analyzed to (1) test strategy switch costs (i.e., poorer performance when participants used different strategies across two consecutive problems than when they used the same strategy) and (2) determine whether strategy switch costs vary with the type of strategies that are executed and/or with the type of problems that are solved. Two series of analyses were run to achieve these ends. First, within-participants ANOVAs with a 2 (problem: small unit, large unit) $\times 2$ (strategy: rounding down, rounding up) $\times 2$ (trial: repeated strategy, unrepeated strategy) designs were run on mean solution latencies and percentages of errors shown by the participants for the second problem on a trial (see Table 1). Second, a series of contrasts were run to test the significance of strategy switch costs in each strategy $\times$ problem condition. For all the results, unless otherwise noted, differences are significant to at least $p<.05$.

The participants were slower when they used different strategies on two consecutive problems $(2,886 \mathrm{msec})$ than when they used the same strategy $(2,828 \mathrm{msec})[F(1,29)=$ $\left.5.47, M S_{\mathrm{e}}=37,150, \eta_{\mathrm{p}}^{2}=.16\right]$. Moreover, they were faster when using the rounding-down strategy than when using the rounding-up strategy $[2,334$ vs. $3,381 \mathrm{msec}$; $\left.F(1,29)=164.08, M S_{\mathrm{e}}=401,003, \eta_{\mathrm{p}}^{2}=.85\right]$ and were faster on large-unit than on small-unit problems [2,796 vs. $\left.2,919 \mathrm{msec} ; F(1,29)=13.99, M S_{\mathrm{e}}=64,828, \eta_{\mathrm{p}}^{2}=.33\right]$. Most important, several interactions were significant [strategy $\times$ problem, $F(1,29)=8.03, M S_{\mathrm{e}}=40,249, \eta_{\mathrm{p}}^{2}=$ .22 ; strategy $\times$ trial, $F(1,29)=47.25, M S_{\mathrm{e}}=73,712$, $\eta_{\mathrm{p}}^{2}=.62 ;$ problem $\times$ trial, $F(1,29)=8.89, M S_{\mathrm{e}}=32,048$, $\eta_{\mathrm{p}}^{2}=.24$; and strategy $\times$ problem $\times$ trial, $F(1,29)=$ $\left.26.94, M S_{\mathrm{e}}=30,215, \eta_{\mathrm{p}}^{2}=.48\right]$. These significant effects showed that (1) the participants were equally fast on small- and large-unit problems [2,358 vs. 2,309 msec; $F(1,29)=2.27$, n.s.] when they used the rounding-down strategy but were faster on large-unit problems than on small-unit problems $[3,283$ vs. $3,479 \mathrm{msec} ; F(1,29)=$ 16.15] when they used the rounding-up strategy; (2) the participants were slower on unrepeated-strategy trials than on repeated-strategy trials when they solved smallunit problems $[2,855$ vs. $2,982 \mathrm{msec} ; F(1,29)=10.86]$ but were equally fast when they solved large-unit problems $(2,801$ vs. $2,790 \mathrm{msec} ; F<1)$; and (3) the participants were slower on unrepeated-strategy trials than on repeated-strategy trials when they used the rounding-down strategy [2,483 vs. $2,184 \mathrm{msec} ; F(1,29)=50.67]$ but were faster when they used the rounding-up strategy $[3,289 \mathrm{vs}$. $3,472 \mathrm{msec} ; F(1,29)=17.61]$.

The participants made very few errors (i.e., percentages of trials on which they used a strategy different from the required strategy). Error rates were larger when the participants solved small-unit problems than when they solved large-unit problems $[1.03 \%$ vs. $0.58 \% ; F(1,29)=7.86$, $\left.M S_{\mathrm{e}}=1.51, \eta_{\mathrm{p}}^{2}=.21\right]$, when they used the rounding-up strategy than when they used the rounding-down strategy $\left[1.03 \%\right.$ vs. $0.58 \% ; F(1,29)=5.21, M S_{\mathrm{e}}=2.27, \eta_{\mathrm{p}}^{2}=$ .15], and on unrepeated-strategy trials than on repeatedstrategy trials $\left[1.03 \%\right.$ vs. $0.61 \% ; F(1,29)=7.86, M S_{\mathrm{e}}=$ $\left.1.51, \eta_{\mathrm{p}}^{2}=.18\right]$.

In sum, strategy switch costs for strategy execution are the most important and original findings in Experiment 1 . The participants showed poorer performance on a given trial when they executed different strategies on two consecutive trials than when they executed the same strategy. Such strategy switch costs were found here when the participants used the easy, rounding-down strategy and solved the easy small-unit problems.

Table 1

Mean Solution Latencies (in Milliseconds) and Percentages of Errors on the Second Problem of a Trial As a Function of Strategy and Problem Transition (Experiment 1)

\begin{tabular}{cccccccc}
\hline \multirow{2}{*}{ Problems } & \multicolumn{3}{c}{ Rounding-Down Strategy } & & \multicolumn{3}{c}{ Rounding-Up Strategy } \\
\cline { 2 - 4 } & Repeated & Unrepeated & SSC & & Repeated & Unrepeated & SSC \\
\hline Small-unit problems & 2,116 & 2,601 & $485^{*}$ & & 3,595 & 3,363 & $-231^{*}$ \\
Large-unit problems & 2,251 & 2,364 & $112^{*}$ & & 3,351 & 3,214 & $-137^{*}$ \\
$M$ & 2,184 & 2,482 & $298^{*}$ & & 3,473 & 3,289 & $-184^{*}$ \\
& \multicolumn{7}{c}{ Solution Latencies } \\
Small-unit problems & 0.33 & 0.89 & 0.56 & & 1.11 & 1.89 & +0.67 \\
Large-unit problems & 0.44 & 0.67 & 0.22 & & 0.56 & 0.67 & +0.11 \\
$M$ & 0.39 & 0.78 & 0.39 & & 0.83 & 1.28 & +0.39 \\
\hline
\end{tabular}

Note-Repeated, repeated-strategy trial; unrepeated, unrepeated-strategy trial; SSC, strategy switch cost (unrepeated - repeated). ${ }^{*} p<.05$. 


\section{EXPERIMENT 2 Control Experiment}

In Experiment 1, solution times were measured by the experimenter's pressing the space bar as soon as possible after the participants' responses. Combined with asking the participants to calculate out loud, such an approach was adopted to make sure that the participants were using the strategy that they were cued to use on each trial. However, this raises the possibility of measurement errors that would make the latency data unreliable, although the experimenter was completely naive regarding the goal of the experiment, the factors that we manipulated, and our hypotheses. It could be argued that such data unreliability may explain some of the findings in Experiment 1, findings such as why strategy switch costs were found only when the participants solved small-unit problems or when they used the rounding-down strategy. The goal of Experiment 2 was to replicate the strategy switch costs found in Experiment 1 while controlling for potential additional variance in the solution times. This was done by measuring latencies directly with a voice key relay that was triggered at the onset of the participants' oral responses.

\section{Method}

Participants. Twenty ( 8 males; mean age, 24 years 1 month; age range, 24.2-28.1) undergraduates from the University of Provence received course credit for their participation.

Stimuli and Procedure. The stimuli and procedure were exactly the same as those in Experiment 1, with two exceptions. First, the participants were not asked to calculate out loud. Second, reaction times were measured with a voice key relay. Thus, the timing of each response began when the problem and the cue appeared on the screen and ended when the participants started to state their answers. The participants were asked to articulate their answers once they had found them and to not calculate out loud, so that the voice key would not be triggered while products were being estimated.

\section{Results}

Of the data, $1.8 \%$ were dropped because of failure or inappropriate recording of timing with the voice key (e.g., the participants' coughing or speaking out loud before finding an estimate). The same $2 \times 2 \times 2$ (problem $\times$ strategy $\times$ trial) ANOVA designs as those in Experiment 1 were used to analyze the participants' performance (see
Table 2 for means). The participants were slower when they used different strategies on two consecutive problems $(2,568 \mathrm{msec})$ than when they used the same strategy $(2,487 \mathrm{msec})\left[F(1,19)=6.86, M S_{\mathrm{e}}=38,677, \eta_{\mathrm{p}}^{2}=.27\right]$. Moreover, they were faster when using the rounding-down strategy than when using the rounding-up strategy $[2,118$ vs. 2,937 msec; $F(1,19)=73.49, M S_{\mathrm{e}}=364,883, \eta_{\mathrm{p}}^{2}=$ .79]. Most important, the strategy $\times$ trial $[F(1,19)=$ 11.79, $\left.M S_{\mathrm{e}}=25,569, \eta_{\mathrm{p}}^{2}=.38\right]$ and problem $\times$ strategy $\times$ trial $\left[F(1,19)=5.03, M S_{\mathrm{e}}=73,264, \eta_{\mathrm{p}}^{2}=.21\right]$ interactions were significant. The participants were slower on unrepeated-strategy trials than on repeated-strategy trials when they used the rounding-down strategy $(2,282 \mathrm{vs}$. $2,034 \mathrm{msec} ; F(1,19)=18.77$ ] but were equally fast when they used the rounding-up strategy $(2,934 \mathrm{vs.} 2,940 \mathrm{msec}$; $F<1)$ This was the case when the participants solved small-unit problems. The participants made very few errors, and they erred more when they used the rounding-up strategy than when they used the rounding-down strategy $\left[4.5 \%\right.$ vs. $1.6 \% ; F(1,19)=13.05, M S_{\mathrm{e}}=25.51, \eta_{\mathrm{p}}^{2}=$ .41]. No other effects were significant.

In sum, Experiment 2 replicated the most important findings from Experiment 1 regarding strategy switch costs. The participants obtained better performance on a given trial when they executed the same strategy on that trial and on the preceding trial. Such strategy switch costs interacted with strategy and problem difficulty, since they were found when the participants used the rounding-down strategy to solve small-unit problems. These strategy switch costs, like those in Experiment 1, were observed in a situation in which the participants were told which strategy to use on a given problem. Would they be observed when they selected among available strategies? Data from the task-switching literature suggest that this should be the case, since task switch costs have also been observed in voluntary task switching (Arrington \& Logan, 2004, 2005). Experiment 3 tested strategy switch costs when the participants were free to choose among strategies.

\section{EXPERIMENT 3 Strategy Switch Costs in Strategy Selection}

In this experiment, we tested strategy switch costs during strategy selection in the context of two-digit addition

Table 2

Mean Solution Latencies (in Milliseconds) and Percentages of Errors on the Second Problem of a Trial As a Function of Strategy and Problem Transition (Experiment 2)

\begin{tabular}{|c|c|c|c|c|c|c|}
\hline \multirow[b]{2}{*}{ Problems } & \multicolumn{3}{|c|}{ Rounding-Down Strategy } & \multicolumn{3}{|c|}{ Rounding-Up Strategy } \\
\hline & Repeated & Unrepeated & $\mathrm{SSC}$ & Repeated & Unrepeated & SSC \\
\hline \multicolumn{7}{|c|}{ Solution Latencies } \\
\hline Small-unit problems & 2,006 & 2,282 & $276^{*}$ & 3,042 & 2,952 & -90 \\
\hline Large-unit problems & 2,062 & 2,122 & 60 & 2,838 & 1,916 & 79 \\
\hline$M$ & 2,034 & 2,202 & $168^{*}$ & 2,940 & 2,934 & -5 \\
\hline \multicolumn{7}{|c|}{ Percent Errors } \\
\hline Small-unit problems & 0.9 & 3.0 & $2.1^{*}$ & 4.3 & 3.8 & -0.5 \\
\hline Large-unit problems & 1.2 & 1.2 & 0.0 & 5.3 & 4.5 & -0.8 \\
\hline$M$ & 1.1 & 2.1 & $1.0^{*}$ & 4.81 & 4.1 & -0.7 \\
\hline
\end{tabular}

Note-Repeated, repeated-strategy trial; unrepeated, unrepeated-strategy trial; SSC, strategy switch cost (unrepeated - repeated). ${ }^{*} p<.05$. 
problem solving. We tested another task and two other strategies for several reasons. First, we wanted to replicate the strategy switch costs found in Experiment 1 and to generalize them to another task and strategy context. Moreover, previous studies have documented in great detail the strategies that participants use while solving two-digit addition problems. In particular, these previous works showed that the two strategies that participants use spontaneously are of equal level of difficulty and yield comparable speed and accuracy performance (e.g., Beishuizen, 1993; Beishuizen, Van Putten, \& Van Mulken, 1997; Lemaire \& Arnaud, 2008; Lucangeli, Tressoldi, Bendotti, Bonanomi, \& Siegel, 2003). This was of interest here since it offers a case in which the relative difficulty of strategies can be controlled so as to determine whether strategy switch costs are driven by this factor, as was suggested by the findings from Experiments 1 and 2. Finally, previous works showed that solving two-digit addition problems takes longer than accomplishing computational estimation tasks. For example, Lemaire and Calliès (2009) found that participants took $5.9 \mathrm{sec}$ to solve two-digit addition problems with the two strategies tested here (vs. $2.9 \mathrm{sec}$ in the computational estimation task in Experiment 1). Moreover, in contrast to Experiments 1 and 2, the two strategies tested here led to the same answer. It was of interest to determine whether participants' performance is influenced by strategy switch costs in such contexts. Indeed, it is possible that given the magnitude of strategy switch costs, relative to overall performance, strategy switch costs are not observed when time to select and execute a given strategy is too long.

In Experiment 3, the participants saw 80 trials. A trial included two successive two-digit addition problems and a series of letters. The participants were told which strategy (full- or partial-decomposition strategy) to use on the first problem and could choose among these two strategies on the second problem. In the full-decomposition strategy, the participants first added decades, then units, and finally the two results (e.g., $34+53 ; 30+50=80 ; 4+3=7$; $80+7=87$ ). In the partial-decomposition strategy, the participants first added decades of the second operand to the first operand and then units (e.g., $34+53 ; 34+50=$ $84+3=87$ ).

Strategy switch costs in strategy use would be seen if using a strategy on a given problem influenced the strategy that we chose on the following problem. This predicts that mean percentage of use of partial decomposition on the second problem of each trial should be larger if the participants used partial decomposition on the preceding trial than if they used full decomposition. Similarly, mean percentage of use of full decomposition on the second problem of each trial should be larger if the participants used it on the preceding trial. In other words, mean percentage of repetitions of the same strategy across two consecutive problems should be significantly different from chance. Such an outcome would result from the fact that the participants did not completely inhibit a just-executed strategy when choosing among available strategies to solve a new problem.
Regarding strategy execution, we expected to replicate the strategy switch costs found in Experiment 1. That is, the participants should be slower and less accurate when they executed different strategies on two consecutive problems of a trial than when they executed the same strategy on each problem. More specifically, the participants should have poorer performance when they executed the partialdecomposition strategy after using the full-decomposition strategy than after executing the partial-decomposition strategy on the preceding trial. Similarly, they should be slower and less accurate to execute the full-decomposition strategy after executing the partial decomposition strategy on the preceding trial than after executing the fulldecomposition strategy.

\section{Method}

Participants. Twenty-five (6 males; mean age, 21 years 3 months; age range, 17.11-29.9) undergraduates from the University of Provence received course credit for their participation.

Stimuli and Design. Each participant completed 80 trials. Each trial was made of a first two-digit addition problem followed by a second two-digit addition problem. This second problem was followed by a series of five letters. Half of the five-letter series included only consonants or only vowels, and half included both types of letters. Half of the choice and no-choice problems had a carry on the unit digit (e.g., $28+54$ ), and half were no-carry problems (e.g., $62+23$ ). Carry and no-carry problems were matched on size of correct sums. If the first problem of a trial had a carry, half of the second problems had a carry, and half were no-carry problems. Similarly, if the first problem had no carry, half of the second problems had a carry, and half were no-carry problems. Moreover, (1) no operands had a 0 unit digit (e.g., $40+82$ ), (2) no operands had a 5 unit digit (e.g., $45+82)$, (3) no digits were repeated within operands (e.g., $44+82),(4)$ no reverse orders of operands were used (e.g., $43+82$ and $82+43)$, (5) the first operand was larger than the second in half the carry and no-carry problems and smaller in the other half, and (6) no operand had its closest decade equal to 0,10 , or 100.

Procedure. The participants were individually tested in one session that lasted approximately $45-60 \mathrm{~min}$. First, each strategy was explained, illustrated, and practiced on three carry and three no-carry problems. To use the full-decomposition strategy, the participants were told to first add decades, then units, and finally the two results. For example, to solve $34+53$, they were asked to do $30+50=$ $80,4+3=7$, and $80+7=87$. To use the partial-decomposition strategy, the participants first added decades of the second operand to the first operand and then units. For $34+53$, they did $34+$ $50=84+3=87$. After this initial practice period, no participants had difficulties with either strategy. Then the participants were told that they would see 80 trials and that each trial would include one two-digit addition problem, followed by another two-digit addition problem, followed by a series of five letters.

The participants were told that the first problem would appear with either "DC" (for décomposition complète, or full decomposition in French), indicating that they should use the full-decomposition strategy to solve the current problem, or "DP" (for décomposition partielle, or partial decomposition in French), indicating that they should use the partial-decomposition strategy on the current problem. To solve the second problem, the participants were informed that they were free to choose between the partial-decomposition and the full-decomposition strategies. After the second problem, we asked them to tell us which of the two strategies they had used to solve this problem.

The experimental problems were presented in 48-point bold Courier font (black color) in the center of a 14-in. computer screen controlled by a Sony G-FX201 PC. Each trial began with a blank screen for $1,500 \mathrm{msec}$, followed by a warning signal (*) appearing 
for $400 \mathrm{msec}$ in the center of the screen. The first problem of a trial and the cue were then simultaneously displayed on the computer screen. The cue appeared $2 \mathrm{~cm}$ below the problem and, together with the problem, remained on the screen until the participants' response. The cue (font, bold Courier; size, 48) was "?" for the second problem on a trial. The second problem on a trial was presented $1,000 \mathrm{msec}$ after participants' response to the first problem. Timing of each response began when the problem and the cue appeared on the screen and ended when the experimenter pressed the space bar of the computer keyboard, the latter event occurring as soon as possible after the participants' responses. A blank screen followed the participants' responses for $1,000 \mathrm{msec}$. An "* " followed the blank screen and was presented for $400 \mathrm{msec}$. Finally, the five-letter stimulus was displayed on the screen until the participants determined whether it contained only vowels, only consonants, or both types of letters. The participants were asked to solve arithmetic problems aloud as a control showing that they were using the strategy that they had been cued to use or that they claimed to have used (which they did on $100 \%$ of the problems). The experiment was controlled by E-Prime software.

\section{Results}

The results were analyzed to determine whether using a strategy on a given trial influences strategy use and strategy execution on the subsequent trial. To determine whether the participants tended to repeat the same strategy over two consecutive trials more often than to use different strategies, we analyzed mean percentages of repetitions (i.e., percentages of trials on which the participants used the same strategy for the two problems on each trial). Overall, the participants tended to repeat the same strategy over two consecutive trials more often than they changed strategy [mean percentage of repetitions: $59 \% ; t(24)=3.96$ ]. Mean percentages of strategy repetitions on a given trial (see Figure 1) were analyzed with a within-participants ANOVA involving a 2 (strategy: full decomposition or partial decomposition) $\times 2$ (carry on the second problem: present or absent) design. They tended to repeat the same strategy more often when the target problem was a no-carry problem than when it was a carry problem $[62 \%$ vs. $\left.55 \% ; F(1,24)=10.67, M S_{\mathrm{e}}=98.96, \eta_{\mathrm{p}}^{2}=.31\right]$. The effect of strategy and the strategy $\times$ carry interaction were not significant $(F \mathrm{~s}<1)$.

Regarding strategy execution, mean solution latencies and percentages of errors for the second problem on a trial were analyzed with within-participants ANOVAs involving

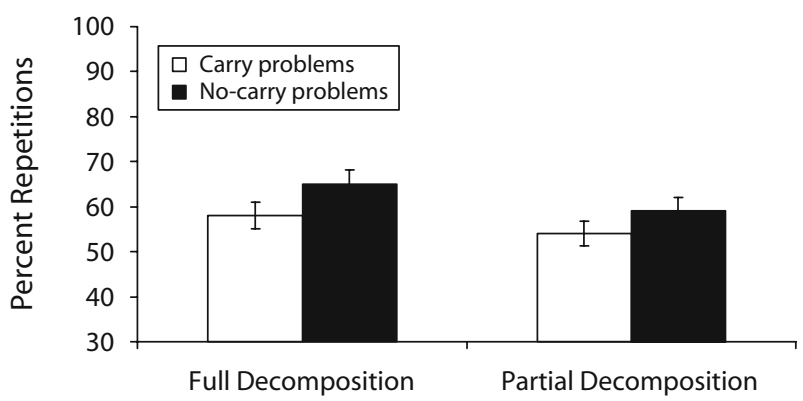

Figure 1. Mean percentages of repetitions of the same strategy across two successive problems for full-decomposition and partial-decomposition strategies while carry and no-carry problems were solved (Experiment 3). a 2 (trial: repeated or unrepeated strategy) $\times 2$ (strategy: full decomposition or partial decomposition) $\times 2$ (carry: present or absent) design. Means are presented in Table 3. Analyses of solution latencies showed that the participants were slower when they used different strategies on two consecutive trials than when they repeated the same strategy $\left[8,664\right.$ vs. $8,465 \mathrm{msec} ; F(1,24)=12.9, M S_{\mathrm{e}}=$ $\left.1,538,885, \eta_{\mathrm{p}}^{2}=.25\right]$ and when they solved carry problems than when they solved no-carry problems $[9,667$ vs. $7,463 \mathrm{msec} ; F(1,24)=58.67, M S_{\mathrm{e}}=4,139,463, \eta_{\mathrm{p}}^{2}=$ .71]. Although the carry $\times$ trial interaction was not significant $\left[F(1,24)=2.05, M S_{\mathrm{e}}=1,821,876, \eta_{\mathrm{p}}^{2}=.08\right]$, strategy switch costs were significant for carry problems $[473 \mathrm{msec} ; F(1,24)=5.86]$ but nonsignificant for nocarry problems $(F<1)$. Although error rates were low, the participants made more errors on carry problems than on no-carry problems $[9.5 \%$ vs. $3.1 \% ; F(1,24)=24.10$, $\left.M S_{\mathrm{e}}=86.48, \eta_{\mathrm{p}}^{2}=.50\right]$. Mean percentages of incorrectly solved problems were comparable on repeated-strategy and unrepeated-strategy trials $(6.6 \%$ vs. $6.1 \% ; F<1)$. The strategy $\times$ trial interaction was significant $[F(1,24)=$ $\left.4.61, M S_{\mathrm{e}}=57.32, \eta_{\mathrm{p}}^{2}=.16\right]$. Although strategy switch costs were nonsignificant for each strategy, this resulted from the participants' tending to err more on unrepeatedstrategy trials than on repeated-strategy trials when they used the partial-decomposition strategy $(8.2 \%$ vs. $6.4 \%$; $F<1)$ and to do the reverse when they used the fulldecomposition strategy $[3.9 \%$ vs. $6.7 \% ; F(1,24)=3.43$, $p=.08]$. No other effects were significant for either solution time or errors.

In sum, strategy switch costs were seen in both strategy use and strategy execution. The participants tended to repeat the same strategy over two consecutive trials (and even more so for no-carry problems than for carry problems). They also had poorer performance when they used different strategies than when they used the same strategy. Such strategy switch costs were found for both the fulland partial-decomposition strategies.

\section{GENERAL DISCUSSION}

These experiments have shown strategy switch costs (see Figure 2). The participants obtained poorer performance when they used different strategies on two consecutive trials than when they used the same strategy. They also tended to use the same strategy over two consecutive problems more often than using different strategies. Such strategy switch costs in strategy execution and strategy selection were found when the participants executed the easiest strategy and when they solved easy problems. These findings have implications for furthering our understanding of strategy choices and strategy execution. We will discuss first the processes possibly underlying switch costs and then the implications of these switch costs on models of strategy choices.

Why did the participants obtain poorer performance when they used different strategies than when they repeated the same strategy on two consecutive trials, and why did they tend to select the same strategy on two consecutive problems? Since the strategy switch costs found 
Table 3

Mean Solution Latencies (in Milliseconds) and Percentages of Errors on the Second Problem of a Trial When Participants Used the Full- and Partial-Decomposition Strategies on Repeated-Strategy and Unrepeated-Strategy Trials (Experiment 3)

\begin{tabular}{|c|c|c|c|c|c|c|}
\hline \multirow[b]{2}{*}{ Problems } & \multicolumn{3}{|c|}{ Full-Decomposition Strategy } & \multicolumn{3}{|c|}{ Partial-Decomposition Strategy } \\
\hline & Repeated & Unrepeated & SSC & Repeated & Unrepeated & SSC \\
\hline \multicolumn{7}{|c|}{ Solution Latencies } \\
\hline No-carry problems & 7,341 & 7,570 & $229^{*}$ & 7,112 & 7,828 & $716^{*}$ \\
\hline Carry problems & 9,868 & 9,897 & 29 & 9,540 & 9,362 & -178 \\
\hline$M$ & 8,604 & 8,733 & 129 & 8,326 & 8,595 & 269 \\
\hline \multicolumn{7}{|c|}{ Percent Errors } \\
\hline No-carry problems & 2.95 & 1.22 & -1.73 & 3.67 & 4.47 & 0.80 \\
\hline Carry problems & 10.52 & 6.67 & -3.85 & 9.06 & 11.89 & 2.83 \\
\hline M & 6.73 & 3.95 & -2.78 & 6.37 & 8.18 & 1.81 \\
\hline
\end{tabular}

Note-Repeated, repeated-strategy trial; unrepeated, unrepeated-strategy trial; SSC, strategy switch cost (unrepeated - repeated). ${ }^{*} p<.05$.

here bear some similarities with the task switch costs reported in the task switch cost literature, strategy switch costs may involve the same mechanisms that have been proposed to account for task switch costs. Thus, two types of mechanisms can be envisaged to account for strategy switch costs: control executive and/or priming processes.

Priming processes may underlie strategy switch costs. Such a priming hypothesis (or strategy set inertia) has also been proposed in the task-switching literature (e.g., Allport \& Wylie, 2000). In the case of strategies, this means that recent retrieval and execution of a strategy would influence subsequent availability for retrieval and execution of that strategy. That is, having just executed a strategy to solve a problem makes this strategy more available for retrieval and/or execution while solving the next problem, since this strategy is in a high state of activation. When participants are executing a strategy, if this strategy has just been used, the procedures of this strategy are still activated and, as a consequence, more quickly executed in the repeated-strategy condition. This leads participants to be faster in the repeated-strategy condition. Such facilitative effects of strategy procedure preactivation cannot be found in the unrepeated-strategy condition, because the strategies used on both of the consecutive problems are different. In the unrepeated-strategy condition, persisting involuntary activation from a just-executed strategy interferes with performance on the currently relevant strategy. Participants have to overcome interference from the previous strategy. In other words, priming processes would explain how speed increases in the repeated-strategy condition and decreases in the unrepeated-strategy condition.

Alternatively, like task switch costs (Monsell, 2003, 2005), strategy switch costs can be interpreted as resulting from strategy set reconfiguration processes. That is, once a problem has been solved with a given strategy, the cognitive system must be reconfigured to solve a problem with another (or the same) strategy. Such a strategy set reconfiguration involves a series of executive control processes, such as shifting attention (from the just-executed strategy to the new strategy); retrieving task goals, rules, or procedures (of the new strategy); and activating, executing, and ordering component procedures of the to-be-executed strategy, as well as inhibiting irrelevant strategies (such as the just-executed strategies). In other words, once a problem has been solved with a given strategy, participants must disengage from the just-executed strategy or inhibit activated procedures of this now-irrelevant strategy and must make other strategies available in working memory or activate procedures of the relevant, to-be-executed strategy. The extra time required by unrepeated-strategy trials can be interpreted as the duration of these reconfiguration processes. Given the cognitive costs incurred by such strategy set reconfiguration processes, participants tend to use the same strategy over two (or more) successive trials (as in Experiment 3), unless the benefits of using an alternative strategy outweigh the costs of strategy switching. Our data do not rule out either the priming or the strategy set reconfiguration accounts. It is possible that a number of processes contribute to successful strategy switching, including strategy priming and strategy set reconfiguration processes. Such multiple processes would facilitate performance for repeated-strategy conditions and decrease performance for unrepeated-strategy conditions.

Interestingly, the present strategy switch costs interacted with problem and strategy characteristics. In Experiment 1, strategy switch costs were found only for the easy, small-unit problems and for the easy, rounding-down strategy. In Experiment 2, when reaction times were collected directly, strategy switch costs were found when the participants switched from the hard (rounding-up) strategy to the easy (rounding-down) strategy to solve easy, small-unit problems. In Experiment 3, strategy switch costs were found for no-carry problems. Strategy switch costs were larger for easier, no-carry problems during strategy selection and tended to be larger on these problems during strategy execution. Moreover, interestingly, when strategy difficulty was controlled in Experiment 3, strategy switch costs were found for both partial- and fulldecomposition strategies and were of comparable magnitude. The interaction of strategy switch costs with strategies bears great similarity with the asymmetry of task switch costs. Task switch costs are larger for easy than for hard tasks (e.g., Allport et al., 1994; Yeung \& Monsell, 2003). However, note that task switch costs are sig- 


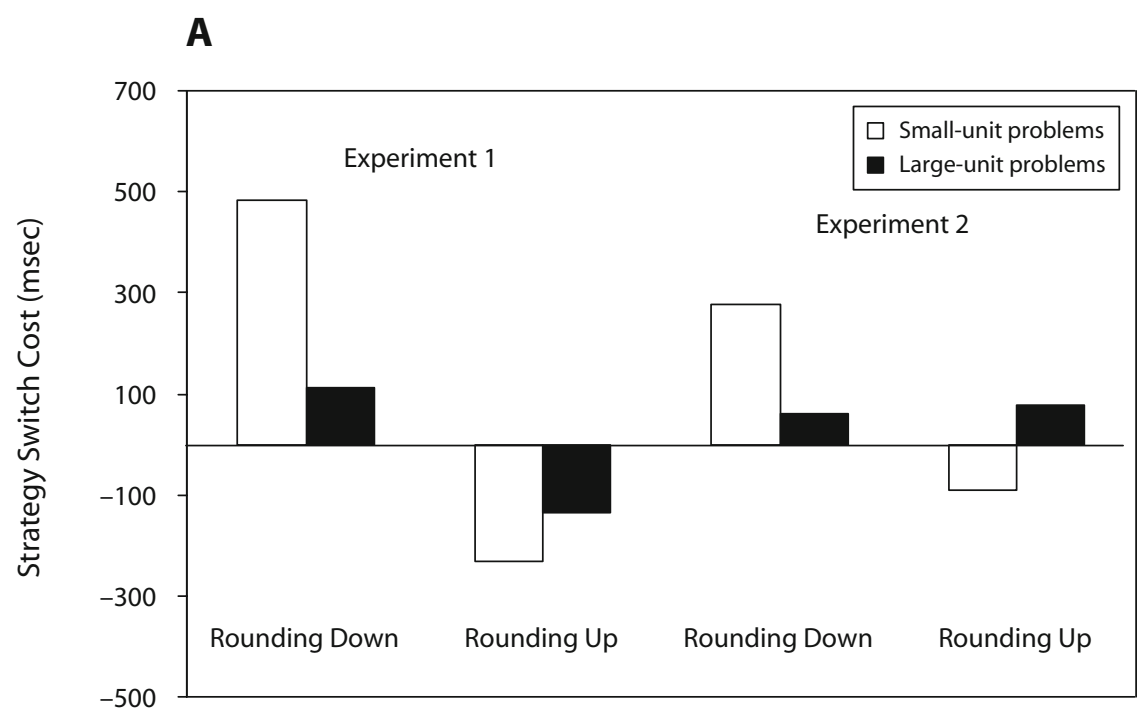

B

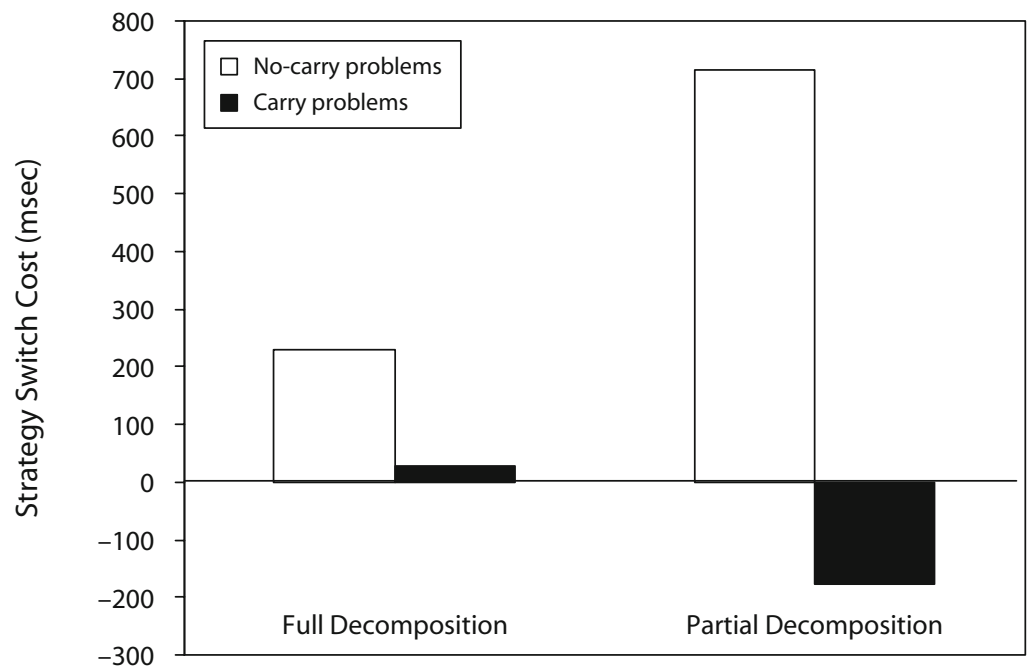

Figure 2. Summary of switch costs (reaction times [RTs] for unrepeated trials - RTs for repeated trials) in each of the three experiments. (A) Experiments 1 and 2. (B) Experiment 3.

nificant when participants switch from an easy to a hard task. Here, strategy switch costs were found only when the participants switched from the hard to the easy strategy during strategy execution (Experiments 1-3) and for both easy and hard problems during strategy selection (Experiment 3). Future studies should replicate the present strategy switch costs during strategy execution in order to determine whether the present strategy switch costs are only a particular variant of the asymmetry of task switch costs or whether strategy switch costs are different from task switch costs in the sense that they are found only when participants switch from a hard to an easy strategy.

To further our understanding of strategy switch costs, future studies should pursue two additional goals in order to replicate this phenomenon and generalize it to domains that are known to show strategic variations (e.g., episodic memory; Dunlosky \& Hertzog, 2001). First, conditions of the occurrence of strategy switch costs should be established. This could be done by examining variations in the magnitudes of strategy switch costs as a function of a number of factors (e.g., proportions of strategy-repeat trials; number of strategies to switch among; interstimulus interval). This would enable us to further our understanding of the boundary conditions under which strategy switch costs are observed and of the underlying processes. Second, future studies should determine whether strategy switch costs may account for still unexplained strategy selection phenomena. For example, relative strategy efficacy never fully accounts for strategy choices, since participants continue to use a strategy when an alternative strategy is slightly faster and more accurate (e.g., Siegler \& Lemaire, 1997). It is possible that, on some items, strategy 
set reconfiguration and/or priming processes influence strategy choices more strongly than (or in combination with) considering relative strategy efficiency and problem features. Experiment 3 suggests that strategy switch costs would promote strategy repetitions to avoid switch costs. The role of strategy switch costs in strategy choices could be investigated by examining how strategy switch costs correlate with participants' use of the best strategy on each item, as well as with participants' characteristics that are known to influence strategy choices (e.g., participants' age, working memory capacities, and arithmetic skills).

The strategy switch costs found here point to the need to elaborate existing theories of strategy selection. Models such as SCADS* (Siegler \& Arraya, 2005), ACT-R (Lovett \& Anderson, 1996), the adaptive decision maker (Payne et al., 1993), RCCL (Lovett \& Schunn, 1999), and SSL (Rieskamp \& Otto, 2006) share several core assumptions concerning strategy selection processes. None of the models assume that strategy choices on a given trial are influenced by using and executing a strategy on a previous trial. Such an assumption could be included in all models of strategy choices.

Moreover, none of the models assume that strategy choices involve strategy set reconfiguration processes. If future studies establish that the difference between repeated-strategy and unrepeated-strategy items found here happens to result in part from strategy set reconfiguration processes and/or priming processes, formal models of strategy choices, such as SSL, SCADS*, ACT-R, and RCCL, could be elaborated to include such strategy set reconfiguration processes. For example, the SCADS* model assumes that strategy choices involve a controlled attention mechanism. In its current version, SCADS* assumes that this mechanism enables the cognitive system to selectively encode problem features, to shift attention from one operand to another during arithmetic problem solving, to interrupt the execution of a strategy while solving a problem, and to select a new strategy for this problem. SCADS* can be extended to assume that controlled attention also enables shifting from one strategy to another on each problem and that this shifting involves strategy set reconfiguration and/or priming processes. Such extensions could also be envisaged for the other computational models of strategy selection (ACT-R, SSL, and RCCL).

\section{AUTHOR NOTE}

This research was supported in part by the CNRS (French NSF) and by a grant from the Agence Nationale de la Recherche (BLAN071_196867). Correspondence about this article should be directed to P. Lemaire, CNRS and Université de Provence, 3 Place Victor Hugo, Case D, 13331 Marseille, France (e-mail: patrick.lemaire@univ-provence.fr).

\section{REFERENCES}

Allport, A., Styles, E. A., \& Hsieh, S. (1994). Shifting attentional set: Exploring the dynamic control of tasks. In C. Umiltà \& M. Moscovitch (Eds.), Attention and performance XV: Conscious and nonconscious information processing (pp. 421-452). Cambridge, MA: MIT Press, Bradford Books.

Allport, A., \& Wylie, G. (2000). Task-switching, stimulus-response bindings, and negative priming. In S. Monsell \& J. S. Driver (Eds.),
Control of cognitive processes: Attention and performance XVIII (pp. 35-70). Cambridge, MA: MIT Press.

Arrington, C. M., \& LogAn, G. D. (2004). The cost of a voluntary task switch. Psychological Science, 15, 610-615.

Arrington, C. M., \& Logan, G. D. (2005). Voluntary task switching: Chasing the elusive homunculus. Journal of Experimental Psychology: Learning, Memory, \& Cognition, 31, 683-702.

Beishuizen, M. (1993). Mental strategies and materials or models for addition and subtraction up to 100 in Dutch second grades. Journal for Research in Mathematics Education, 24, 295-323.

Beishuizen, M., Van Putten, C. M., \& Van Mulken, F. (1997). Mental arithmetic and strategy use with indirect number problems up to one hundred. Learning \& Instruction, 7, 87-106.

CAMPBell, J. I. D. (ED.) (2005). Handbook of mathematical cognition. New York: Psychology Press.

Campbell, J. I. D., \& Xue, Q. (2001). Cognitive arithmetic across cultures. Journal of Experimental Psychology: General, 130, 299-315.

Dunlosky, J., \& HerTzog, C. (2001). Measuring strategy production during associative learning: The relative utility of concurrent versus retrospective reports. Memory \& Cognition, 29, 247-253.

LeFevre, J. A., Bisanz, J., Daley, K. E., Buffone, L., \& Sadesky, G. S. (1996). Multiple routes to solution of single-digit multiplication problems. Journal of Experimental Psychology: General, 125, 284-306.

LeFevre, J. A., Greenham, S. L., \& Waheed, N. (1993). The development of procedural and conceptual knowledge in computational estimation. Cognition \& Instruction, 11, 95-132.

Lemaire, P., \& Arnaud, L. (2008). Young and older adults' strategies in complex arithmetic. American Journal of Psychology, 121, 1-16.

Lemaire, P., Arnaud, L., \& Lecacheur, M. (2004). Adults' age-related differences in adaptivity of strategy choices: Evidence from computational estimation. Psychology \& Aging, 10, 467-481.

Lemaire, P., \& Calliès, S. (2009). Children's strategies in complex arithmetic. Journal of Experimental Child Psychology, 103, 49-65.

Lemaire, P., Lecacheur, M., \& Farioli, F. (2000). Children's strategy use in estimation. Canadian Journal of Experimental Psychology, 54, 141-147.

Lemaire, P., \& Siegler, R. S. (1995). Four aspects of strategic change: Contributions to children's learning of multiplication. Journal of Experimental Psychology: General, 124, 83-97.

Lovett, M. C., \& ANDERson, J. R. (1996). History of success and current context in problem solving: Combined influences on operator selection. Cognitive Psychology, 31, 168-217.

LovetT, M. C., \& SchunN, C. D. (1999). Task representations, strategy variability, and base-rate neglect. Journal of Experimental Psychology: General, 128, 107-130.

Lucangeli, D., Tressoldi, P. E., Bendotti, M., Bonanomi, M., \& SIEGEL, L. S. (2003). Effective strategies for mental and written arithmetic calculation from the third to the fifth grade. Educational Psychology, 23, 507-520

Luchins, A. S. (1942). Mechanization in problem solving. Psychological Monographs, 54, 248.

Luwel, K., Verschaffel, L., Onghena, P., \& De Corte, E. (2003). Flexibility in strategy use: Adaptation of numerosity judgement strategies to task characteristics. European Journal of Cognitive Psychology, 15, 246-266.

MEIRAN, N. (1996). Reconfiguration of processing mode prior to task performance. Journal of Experimental Psychology: Learning, Memory, \& Cognition, 22, 1423-1442.

Meiran, N., Chorev, Z., \& SaPiR, A. (2000). Component processes in task switching. Cognitive Psychology, 41, 211-253.

Monsell, S. (2003). Task switching. Trends in Cognitive Sciences, 7 , 134-140

Monsell, S. (2005). The chronometrics of task-set control. In J. Duncan, L. Phillips, \& P. McLeod (Eds.), Measuring the mind: Speed, control, and age (pp. 161-190). Oxford: Oxford University Press.

Monsell, S., TAYlor, T. J., \& MurPhy, K. (2001). Naming the color of a word: Is it responses or task sets that compete? Memory \& Cognition, 29, 137-151.

Monsell, S., Yeung, N. P., \& Azuma, R. (2000). Reconfiguration of task-set: Is it easier to switch to the weaker task? Psychological Research, 63, 250-264. 
Payne, J. W., Bettman, J. R., \& Johnson, E. J. (1993). The adaptive decision maker. New York: Cambridge University Press.

RiesKamP, J., \& OTto, P. E. (2006). SSL: A theory of how people learn to select strategies. Journal of Experimental Psychology: General, 135, 207-236.

Rogers, R. D., \& Monsell, S. (1995). Costs of a predictable switch between simple cognitive tasks. Journal of Experimental Psychology: General, 124, 207-231.

Siegler, R. S. (2007). Cognitive variability. Developmental Science, 10, 104-109.

Siegler, R. S., \& Arraya, R. (2005). A computational model of conscious and unconscious strategy discovery. In R. V. Kail (Ed.), Advances in child development and behaviour (pp. 1-42). Oxford: Elsevier.

Siegler, R. S., \& Lemaire, P. (1997). Older and younger adults' strategy choices in multiplication: Testing predictions of ASCM using the choice/no-choice method. Journal of Experimental Psychology: General, 126, 71-92.

SoHN, M.-H., \& ANDERSON, J. R. (2001). Task preparation and task repetition: Two-component model of task switching. Journal of Experimental Psychology: General, 130, 764-778.
SoHn, M.-H., \& CARlson, R. A. (2000). Effects of repetition and foreknowledge in task-set reconfiguration. Journal of Experimental Psychology: Learning, Memory, \& Cognition, 26, 1445-1460.

Wylie, G. [R.], \& Allport, D. A. (2000). Task switching and the measurement of "switch costs." Psychological Research, 63, 212-233.

Wylie, G. R., JaVitT, D. C., \& Foxe, J. J. (2003a). The role of response requirements in task switching: Dissolving the residue. NeuroReport, 15, 1079-1087.

Wylie, G. R., Javitt, D. C., \& Foxe, J. J. (2003b). Task switching: A high-density electrical mapping study. NeuroImage, 20, 2322-2342.

Wylie, G. R., JavitT, D. C., \& Foxe, J. J. (2006). Jumping the gun: Is effective preparation contingent upon anticipatory activation in taskrelevant neural circuitry? Cerebral Cortex, 16, 394-404.

Yeung, N. P., \& Monsell, S. (2003). Switching between tasks of unequal familiarity: The role of stimulus-attribute and response-set selection. Journal of Experimental Psychology: Human Perception \& Performance, 29, 455-469.

(Manuscript received February 16, 2009; revision accepted for publication September 23, 2009.) 